\title{
La normalización lingüística de la lengua de signos española
}

\section{The linguistic normalization of the Spanish sign language}

\section{Palabras clave}

Lengua de signos, lingüística, signolingüística, normalización lingüística, política y planificación lingüística.

\section{Keywords}

Sign language, linguistics, sign linguistics, linguistic normalization, politics and linguistic planning.

\section{Introducción}

Hoy en día las personas sordas signantes podemos estar muy satisfechas y orgullosas del punto en el que nos encontramos tras el largo camino recorrido por el reconocimiento de nuestra lengua, la lengua de signos. Andadura que comenzó allá por los años 60 con los primeros estudios lingüísticos serios. Desde entonces se ha avanzado a pasos agigantados en la investigación sobre las lenguas de signos, en España y en el mundo, desde el campo de la lingüística comparativa, descriptiva y aplicada. Pero a pesar de existir actualmente una considerable bibliografía al respecto, todos seguimos coincidiendo en señalar que esta carrera de fondo está aún muy lejos de llegar a su fin. La lengua de signos española (LSE) es una lengua viva, y como reseñaba Ferdinand de Saussure en su Curso de Lingüística General, como lengua siempre estará, por tanto, en evolución. Por ello, aún subsisten demasiadas lagunas, demasiadas incógnitas, demasiadas incongruencias y puntos de vista dispares, lingüísticamente hablando.

Afortunadamente, lejos quedan ya los tiempos en los que las lenguas de signos eran ignoradas por los lingüistas y por la sociedad oyente

\section{Francisco Vera Villaverde} <docente.lse@hotmail.com>

Centro Superior de Lenguas Modernas (CSLM). Universidad de Cádiz.
Para citar:

Vera, F. (2017): "La normalización lingüística de la lengua de signos española”. Revista Española de Discapacidad, 5 (I): 237-243.

Doi: <https://doi.org/IO.5569/23405104.05.0I.I4> 
en general, incluso negada por las leyes de algunos países. $\mathrm{Y}$ es en estos momentos cuando más me acuerdo del artículo de Charles $\mathrm{F}$. Hockett (I960) sobre las propiedades del lenguaje. En él se señalaban las propiedades fundamentales que definen al lenguaje, en tanto sistema para la comunicación y que, a su vez, lo diferencian de otros sistemas de comunicación existentes en el mundo natural. Consideraba una cualidad inexcusable del lenguaje humano, para considerarse como tal: que su medio de transmisión fuera el canal vocal. Esto, hoy en día, no solo a los profesionales que nos dedicamos a la enseñanza e investigación de la lengua de signos, sino a todas las personas sordas, nos suena a 'cachondeo'. Una total imprecisión o al menos una parcialización de los universales lingüísticos hacia la codificación oral de la comunicación. El supuesto de que una lengua deba ser oral para ser considerada tal, ya desde el campo de la lingüística se cae por su propio peso. Pero pensando en positivo, encuentro dos hipótesis para comprender el sentido de esas formulaciones: era una época oscura para las lenguas de signos, así que posiblemente Hockett ni alcanzó a contemplar la comunicación signada en su curso de lingüística moderna y, por otra parte, las personas usuarias de las lenguas de signos reconocemos una descripción fonológica desde la signolingüística, desde la articulación bucal que forma parte de algunos Signos $^{\mathrm{I}}$ (pero no es el caso de matizar en este momento).

Este tipo de afirmaciones categóricas, característico de quienes no están familiarizados con la lengua de signos, han llegado a ocasionar mucho daño, no ya solo a la perspectiva de considerar a las lenguas de signos como lenguas con todas sus propiedades, sino en lo relativo a la educación de las personas sordas, dificultando su desarrollo cognitivo, social,

I. Suscribo y rescato para la redacción de este artículo el criterio de referirse con el término Signo, con mayúscula, para identificar al Signo de las lenguas de signos, y mantener el término signo, en minúscula, para tratar de otros signos como pueden ser las palabras de las lenguas orales entre otros. En: Minguet, A. (coord.) (200I): Signolingüística. Introducción a la lingüística de la lengua de signos española. Valencia: FESORD-CV, p. I I. afectivo, emocional y comunicativo-lingüístico desde edades tempranas. Viendo cómo está el panorama actualmente... ¡cuánto ha llovido desde entonces!

\section{La normalización lingüística de la lengua de signos española}

Me resulta sugerente y atrevido el tema que presento en estas líneas, no exento de polémica entre muchos de mis colegas, sobre el proceso de normalización lingüística de la LSE y las políticas de planificación lingüística que se deben llevar a cabo. Pero sin duda, esta controversia transciende las disputas científicas y académicas y es materia de debate en toda la comunidad usuaria signante.

Todo proceso de normalización de una lengua tiene un bagaje histórico a sus espaldas y la LSE no está exenta de ese bagaje. A lo largo de la historia las lenguas de signos, como lenguas vivas que son, han sufrido transformaciones y han evolucionado con el transcurso del tiempo modeladas fundamentalmente por el uso entre sus usuarios signantes. $\mathrm{Y}$ es gracias a estos usuarios, a esta comunidad lingüística perfectamente definida usuaria de esta lengua minoritaria y minorizada, con una propia identidad cultural, como se ha llegado de forma notoria al reconocimiento de su propia lengua (Díaz Robledo, 2008). Esta comunidad lingüística es plural, totalmente heterogénea, de modo que cada individuo aporta sus propias concepciones sobre la realidad que le rodea. Se trata de concepciones y sistemas de valores individuales que repercuten en el uso de su lengua en interacción con otros hablantes/ signantes, produciéndose en consecuencia cambios significativos en la misma que afectan a todos los niveles lingüísticos, desde el fonológico hasta el pragmático. Por ello, para propiciar su correcto funcionamiento ante todos estos cambios y orientar a sus usuarios hacia un uso correcto de su lengua, llega un momento en el que es necesario tomar medidas, hay que realizar 
un proceso de normalización. Se trata de diseñar una serie de estrategias lingüísticas que aúnen criterios y que proporcionen una estructura sólida que aliente ese correcto funcionamiento (CNLSE, 20I4). Llegados a este punto creo que se está de acuerdo en la necesidad de llevar a cabo este proceso de normalización, es decir, en la necesidad de realizar una planificación orientada a su regulación. Y es aquí cuando se producen las desavenencias; se difiere en el modo en que esto debe llevarse a cabo.

Como en lingüística siempre hay que andarse con pies de plomo no voy a generalizar. ¿Qué entiendo yo por planificación lingüística, por normalización, normativización, estandarización y otros conceptos afines? ¿Cuál sería, desde mi punto de vista, el proceso de normalización que debería llevarse a cabo? ¿Qué ventajas e inconvenientes ofrece este proceso?

De entrada, para poner en valor y resaltar la importancia de lo que aquí trato, me parece interesante mencionar las palabras de Heinz Kloss, lingüista alemán y toda una autoridad internacionalmente reconocida sobre minorías lingüísticas, decía "la intervención sistemática sobre una o más lenguas con ciertos objetivos y estrategias se realiza para modificar la lengua misma (planificación de corpus, normativización) o la utilización de la lengua y su valor social (planificación de estatus, normalización)". Al hilo de esta premisa, sobre la planificación lingüística, coincido en la reflexión que realiza Rosalía Ortega (20I2) en su trabajo de fin de máster sobre el hablante nativo y su papel en la planificación lingüística. Describe la misma como un arma de doble filo que puede usarse como herramienta de liberación o de dominación.

¿Qué entiendo por planificación lingüística? Amén de las numerosas definiciones aportadas por diferentes de autores, concibo la planificación lingüística como un modelo estructurado de estrategias organizadas para sistematizar de manera ordenada los comportamientos lingüísticos de una lengua, buscando soluciones a problemas lingüísticos derivados de su uso y de la propia estructura de la lengua y, de ese modo, conservar la 'pureza' de esa lengua, favoreciendo la unidad y el enriquecimiento de la misma, y garantizando y protegiendo el derecho a hacer un uso correcto de ella.

Dicho esto, se pueden diferenciar dos tipos de planificación lingüística, una funcional y otra formal (Esteban Saiz, 2002). La primera tiene como objetivo cambiar el estatus de la lengua y sus ámbitos de uso (actuaciones para los ámbitos administrativos, la interpretación, la educación, el acceso a la información, etc.), mientras que la formal pretende modificar la lengua en sí y las formas de uso (derecho al uso de la LSE en gestiones ante entidades públicas, programas educativos bilingües, la formación específica de intérpretes de lengua de signos -ILSE- y docentes de LSE, fomento de las actividades culturales, etc.). Esta planificación formal (que es la más interesante a ojos lingüísticos) se centraría en fijar la norma de la lengua, sirviéndose de recursos como diccionarios, corpus léxicos, neologismos, gramática... elementos que son utilizados para recoger Signos que ya existen y aplicar algún grado de normalización sobre ellos, proponer nuevos términos, elaborar una gramática...

Algo en lo que el acuerdo es unánime radica en la necesaria y acertada formulación de una planificación lingüística en el ámbito de LSE, sobre todo debido a su estatus de lengua minoritaria y minorizada. La LSE es una lengua que queda en condiciones de inferioridad y de fuerte influencia con respecto a otras lenguas de su entorno como son el español y las diferentes lenguas autonómicas (Ferreiro Lago y Aroca Fernández, 2008). De este modo, la planificación lingüística se convierte en una estrategia para asegurar que la LSE goce de los mismos derechos y, a la vez, se convierta en una herramienta para luchar contra los obstáculos que dificultan su normalización.

El objetivo de esta planificación puede ser una mera regularización, política o lingüística, que establezca prescripciones sobre el papel a desempeñar por la LSE. Pero no hay que olvidar que las decisiones que se tomen en política lingüística tienen también que estar vinculadas 
al valor simbólico de la misma lengua ya que ésta no cumple sólo una función comunicativa (Marrero Aguiar, 20I2). Esto es así porque las lenguas se utilizan en sociedad y, por lo tanto, los objetivos que a menudo se persiguen (políticos, sociales, económicos...) no pueden eclipsar a los verdaderos protagonistas lingüísticos, sus usuarios y usuarias. Para que una planificación lingüística tenga éxito hay que tener en cuenta a todos esas personas usuarias de su lengua. Muchas veces se ha dicho que una lengua es lo que sus hablantes/signantes quieren que sea, por lo que una planificación que no atienda a las actitudes lingüísticas de los mismos está destinada al fracaso. Las acciones, medidas y decisiones que se tomen y se lleven a cabo en política y planificación lingüística deben tener siempre presente la realidad de su comunidad usuaria.

Por otra parte, esta planificación lingüística debe llevar intrínseco un proceso de normalización, pero ¿qué entiendo yo por normalización lingüística de la LSE? Se trata de un proceso que favorece su regularización. Esta regularización se produciría en dos vertientes: por una parte, mediante el establecimiento de una legislación y de una serie de políticas lingüísticas y, por otra, mediante la normativización lingüística, es decir, mediante la realización del corpus y la norma de la lengua y su variedad estándar por medio de diccionarios, gramáticas de uso, etc. Por lo que para conseguir esa normativización se debe contar con una gramática normativa y una serie de elementos lexicográficos recopilatorios (diccionarios normativos). De este modo se aborda la normalización de la LSE desde dos ámbitos, el ámbito lingüístico y el social. Y trabajando pues sobre estos dos frentes se impulsa tanto la construcción de una gramática como el reconocimiento de la cultura sorda y una planificación apropiada (Vicente Rodríguez, 2004).

Y llegados a este punto nos encontramos con la disputa más apasionada dentro del ámbito de la LSE, englobada dentro de su proceso de planificación lingüística: la tan controvertida estandarización, la conformación de la variedad estándar de la LSE. ¿Qué entiendo yo por estandarización? Una concepción muy extendida entre algunos colegas lingüistas (y no lingüistas) define la variedad estándar como aquella que es aceptada socialmente por sus usuarios signantes y que resulta la más adecuada en contextos formales; otros la definen como el resultado de realizar un compendio de entre las diferentes variedades dialectales, y no faltan los que abogan por una estandarización llevada a cabo bajo criterios de dominación y supremacía negando cualquier posible inclusión de esas variedades dialectales.

Yo entiendo que la lengua de signos escapa al control hasta de los propios signantes, no puede ser controlada aunque sí regulada; entiendo que, por naturaleza, el 'estándar' es artificial y no corresponde al habla de ninguna región concreta. Entiendo que la creación de una lengua de signos estándar representa el triunfo de una cierta variedad funcional, geolectal o sociolectal y que su selección suele ocasionar que otras variedades que difieren de la estándar pierdan su prestigio, generando así un conflicto entre los propios signantes al ser entendida como una actitud excluyente. Por otro lado, también entiendo que se suele asumir que una norma generalizada, aunque necesaria, sea la representación 'real' de la lengua, lo cual creo que no es del todo cierto ya que la lengua no es algo estático sino diverso y cambiante.

Entonces, ¿cómo conformamos la variedad estándar de la LSE? Hay que tener claro que esta variedad estándar tiene que nutrirse y emerger de todas las variedades regionales o conjunto de variedades geográficas, sociales y estilísticas y debe entenderse como una intersección de las mismas. Debe representar el intercambio de toda la comunidad lingüística, legitimada e institucionalizada históricamente. Por ello, pese a su carácter suprarregional debe incluir las aportaciones de los dialectos y sociolectos regionales y normalizarse y transmitirse de acuerdo a criterios unificadores más que secesionistas. Frente a los dialectos y sociolectos, pues, representaría el medio de comunicación más abstracto y de mayor extensión social. Por lo tanto, yo abogaría por el denominado en lingüística como 
modelo de estándar unitario policéntrico (o composicional), es decir, un modelo elaborado por un solo organismo normativo o por un cuerpo coordinado de organismos normativos, en el que se seleccionarían y fusionarían algunas o todas las variedades geográficas de la LSE. La variedad estándar no se basaría sólo en una variedad geográfica de la LSE, sino que incluiría aportaciones de todas las variedades. Aunque bien es cierto que siempre una puede tener más peso que las restantes, teniendo siempre presente que cuando la distancia lingüística entre las variedades geográficas es grande, el modelo de lengua estándar resultante puede resultar artificioso, ya que no es la variedad nativa de nadie, y ello puede dificultar su aceptación por parte de la comunidad lingüística signante.

Otra dificultad a la hora de normalizar y difundir una variedad estándar de la LSE (y de cualquier otra lengua de signos) reside en que la lengua de signos no tiene una forma escrita (es ágrafa). Aunque existen algunos sistemas de transcripción, no cumplen con los requisitos más esenciales para que estos sistemas sean utilizados mediante caracteres gráficos: facilidad de lectura, reproducción, etc. Por tanto, el bilingüismo (español - LSE) surge como la opción que nos queda a la comunidad signante para desempeñar esa función. La LSE quedaría reservada para la comunicación interpersonal, presencial, cara a cara, y el español escrito se sigue usando como medio mayoritario de difusión. Si la estandarización como la supresión de la variabilidad en una lengua es uno de los rasgos más notorios de la LSE, el hecho de carecer de forma escrita hace que proliferen las variedades dialectales.

Creo también importante tener en cuenta, a la hora de realizar la estandarización de la LSE, la necesidad de contar con las aportaciones de aquellas personas signantes que gozan de una mayor 'autoridad' en cuanto al uso de la misma: las personas nativas signantes (sordas u oyentes), con toda la necesaria flexibilidad y prudencia que su condición requiere, ya que los registros que puede ofrecer este colectivo en ocasiones solo son conocidos por un grupo restringido de ellos, debiéndose estos registros de coger con pinzas a la hora de ser incluidos en ese proceso de estandarización. Si solo nos limitáramos a estandarizar lenguas según los registros de este grupo estaríamos conformando una especie de variedad elitista que siempre sería minoritaria. Por lo tanto, no se trata de seguir un criterio basado en la hipoacusia, sino contar con todas las personas usuarias de la LSE por el mismo hecho de ser usuarias, al margen de ser sordas, oyentes, nativas o no nativas. Cualquier aportación siempre debe ser bienvenida; cosa diferente es si finalmente queda recogida o no y, para ello, cualquier proyecto de estandarización debe contar con un nutrido grupo de personas expertas que lleguen a un consenso en base a unos criterios previamente establecidos y que dispongan de los medios y recursos necesarios que garanticen el proceso de estandarización: selección, codificación e implantación de la variedad estándar.

Por último, siempre hay que tener en cuenta que las lenguas crecen, evolucionan y cambian; aparecen nuevos términos que se hacen convencionales con el uso. Uno de los aspectos más destacados de la normalización de una lengua es la elaboración de diccionarios y material lexicográfico con todo lo que ese trabajo y estudio supone. Para llevarlo a cabo debe formarse un grupo de expertos que debatan sobre la inclusión de cada término en base a una serie de criterios consensuados, pero sin olvidar que todas las lenguas tienen un proceso natural de estandarización, y en el caso de las personas sordas signantes ese proceso es más acusado. Valgan como ejemplos notorios los casos de personas sordas que se desplazan a otras regiones con el consecuente intercambio de Signos, tomando conciencia de las nuevas variaciones que allí residen. O como ocurre con la presencia de ILSE en los medios de comunicación (noticiarios), que tienen un rápido efecto estandarizador sobre toda la comunidad signante.

Y por si todas estas dificultades en el camino hacia la normalización lingüística de la LSE no fueran suficientes, ¿qué decir de la influencia del español sobre la LSE?, ¿cómo afecta una lengua oral mayoritaria y dominante como el español al proceso de normalización de la LSE? Bien 
es cierto que siempre han existido contactos entre lenguas porque siempre han existido contactos entre sus diferentes comunidades de hablantes/signantes, produciendo todo tipo de mezclas léxicas, extranjerismos, bilingüismos... elementos que siempre enriquecen sin duda. $\mathrm{Y}$ esto es así porque no hay idiomas que estén completamente aislados. Es inevitable que existan esas 'intromisiones'. Pero quiero avisar: aterrados estamos los más puristas, entre los que me encuentro, y no tan puristas, de la escandalosa injerencia que está teniendo el español sobre la LSE a todos los niveles lingüísticos, sobre todo gramatical y sintáctico. Impotentes y descorazonados estamos por la desmesurada facilidad con la que el español se inmiscuye en el terreno de la LSE, con la nefasta consecuencia de producirse una pérdida en la calidad lingüística de la misma. Y más que por lo que he estado describiendo anteriormente a lo largo de este artículo, es por esto último por lo que más urge la necesidad de realizar una planificación y normalización lingüística.

Quiero dejar claro que los códigos lingüísticos que mezclan la lengua oral y signada, como es el caso del sistema alternativo de comunicación (SAC) denominado bimodal, muy usado en el ámbito educativo con el alumnado sordo, no debe entenderse nunca como una medida de normalización, sino como algo que puede afectar negativamente a la evolución de la LSE como lengua. El traslado de este método SAC de la educación al ámbito lingüístico deteriora significativamente la calidad lingüística de la LSE. Aunque parezca una exageración, no son pocos los casos de desaparición de lenguas minoritarias por injerencias desorbitadas sobre las mismas de lenguas dominantes, teniendo como resultando la completa sustitución de la lengua minoritaria por la dominante. Aunque también no es menos cierto que ha habido casos en los que gracias a la intervención decidida de las instituciones lingüísticas y la toma de conciencia de su comunidad de hablantes han conseguido frenar la extinción de una lengua, impulsando la normalización de ese idioma minorizado.

Si el objetivo último de la normalización lingüística es permitir que las personas signantes españolas hagan un uso adecuado y correcto de la LSE, sería necesario realizar este proceso de normalización mediante una planificación del estatus, como la llevada a cabo por el Centro de Normalización Lingüística de la Lengua de Signos Española (CNLSE); una planificación del corpus, como los trabajos llevados a cabo con el objetivo de difundir una variedad estándar de la LSE teniendo en cuenta el consenso de la comunidad signante mediante una base de datos multimedia: el "Tesoro de la Lengua de Signos Española”, que permite la validación de los datos por los propios usuarios signantes de distintas regiones o provincias y además posibilita la difusión de los Signos recopilados (CNLSE, 20I4); una política lingüística, fomentada desde las instituciones no solo de personas sordas sino administrativas, como corporaciones locales, diputaciones, ministerios..., y un cultivo de la lengua, fomentada por los profesionales educativos. Remarcar que, sobre todo, el mayor esfuerzo en la estandarización de la LSE debe centrarse en la normalización social con la implicación total de su comunidad lingüística signante fomentada por el apoyo de las instituciones que contribuyan a esta normalización social.

Otra forma de paliar esta injerencia del español sobre la LSE consistiría en la elaboración de un corpus que pretenda 'purificar' esa variedad lingüística estándar limitando el uso de los términos denominados extranjerismos, así como realizar una planificación del corpus mediante la modernización del léxico, permitiendo resolver la necesidad de nuevos términos para conceptos tecnológicos, económicos, médicos, políticos, sociales... Muchos autores han lanzado hipótesis sobre la intensidad de los movimientos más puristas en grupos de población que, a lo largo de la historia, han visto atacada su identidad y han padecido estas experiencias adversas. No obstante, a lo largo de la historia de las personas sordas se ha podido comprobar cómo las generaciones más oprimidas han escogido las posturas más radicales en comparación con las nuevas generaciones que han crecido en un ambiente de mayor respeto e integración. 


\section{Referencias bibliográficas}

CNLSE (20I4): La Lengua de Signos Española hoy. Informe de la situación de la lengua de signos española. Actas del Congreso CNLSE sobre la investigación de la lengua de signos española 2013. Madrid: Real Patronato sobre Discapacidad.

Díaz Robledo, C. M. (2008): "Hacia la normalización de la lengua de signos española. El español, lengua de traducción para la cooperación y el diálogo". En Lara, L. F. (presidencia). Ponencia llevada a cabo en el IV Congreso El Español, Lengua de Traducción. Universidad de Castilla-La Mancha, Toledo (en línea). <http://cvc.cervantes.es/lengua/esletra/ pdf/o4/oI7_diaz.pdf>, acceso 28 de noviembre de 2016.

Esteban Saiz, M. L. et al. (2002): "Planificación Lingüística de la Lengua de Signos Española (LSE)". En Gómez Fernández, A. (presidencia). Ponencia llevada a cabo en el III Congreso de la CNSE Un nuevo impulso a la participación. Fundación CNSE, Zaragoza.

Ferreiro Lago E. y Aroca Fernández, E. (2008): "Cambio cultural, normalización lingüística de la lengua de signos española”. En Gabilondo, A. (presidencia). Ponencia llevada a cabo en el VIII Congreso de Lingüística General El valor de la diversidad [meta]lingüística. Fundación CNSE, Madrid (en línea). <http://elvira.lllf. uam.es/clg8/actas/pdf/paperCLG37.pdf>, acceso ro de diciembre de 2016.
Hockett, C. F. (I960): “The Origin of Speech". Scientific American, 203: 88-I II.

Marrero Aguiar, V. (20I2): "La normalización en LSE. Perspectivas desde la Lingüística”. Ponencia llevada a cabo en III Congreso Nacional de Lengua de Signos Española. UNED- CNSE, Madrid.

Minguet, A. (coord.) (200I): Signolingüística. Introducción a la lingüistica de la lengua de signos española. Valencia: FESORD-CV.

Ortega Pegalajar, R. (2012): El hablante nativo y su papel en la planificación lingüistica (Trabajo Fin de Máster). Universidad de Valladolid (en línea). <https:/uvadoc.uva.es/ bitstream/I0324/2776/I/TFM-G\% 2083.pdf>, acceso I de diciembre de 2016 .

Saussure, F. (I99I): Curso de lingüistica general. Madrid: Akal.

Vicente Rodríguez, M. C. et al. (2004): "Lexicografía y estandarización lingüística: el caso de la lengua de signos española". En Gabilondo, A. (presidencia). Ponencia llevada a cabo en el VIII Congreso de Lingüística General El valor de la diversidad [meta]lingüistica. Fundación CNSE, Madrid (en línea). <http:// www.lllf.uam.es/clg8/actas/ActasCLG8.pdf>, acceso io de diciembre de 2016. 